\title{
Abnormal autonomic stress responses in obstructive sleep apnoea are reversed by nasal continuous positive airway pressure
}

\author{
D. Veale*, J.L. Pépin+, B. Wuyam*\#, P.A. Lévy*+\#
}

\begin{abstract}
Abnormal autonomic stress responses in obstructive sleep apnoea are reversed by nasal continuous positive airway pressure. D. Veale, J.L. Pépin, B. Wuyam, P.A. Lévy. CERS Journals Ltd 1996.

ABSTRACT: Obstructive sleep apnoea (OSA) is associated with abnormalities in autonomic stress tests, which are tests of cardiovascular response in the autonomic nervous system (ANS). The level of abnormality has been related to the level of overnight arterial oxygen saturation $\left(\mathrm{S}_{\mathrm{a}}, \mathrm{O}_{2}\right)$. We have studied ANS function preand post-treatment with nasal continuous positive airway pressure (nCPAP) in six males with moderately severe or severe OSA (apnoea/hypopnoea index (AHI) median 51 (range 14-74) events' $h^{-1}$ of sleep).

Tests consisted of heart rate responses to Valsalva manoeuvre, deep breathing, and change of posture from lying to standing. In addition, systolic blood pressure (SBP) response to standing and diastolic blood pressure (DBP) response to handgrip were studied. Each abnormal test (compared to published normal values) scored +1.0 and each marginal test result (90-95\% confidence interval for normals) scored +0.5. A total score was calculated for the five tests performed in the evening and morning (maximum score 10 per patient). Patients had been receiving treatment for more than 1 year (median 471 (389-624) days) and objective compliance was monitored by a clock counter in the nCPAP machines.

Five of six patients had regularly used nCPAP (mean $\left.7.8 \mathrm{~h} \cdot n i g h t^{-1}\right)$ and all showed a normalization in ANS test score: pre-nCPAP $2(1-4.5)$, post-nCPAP $0.2(0-0.5)$ $(\mathrm{p}<0.05$, Wilcoxon signed rank test). One poorly compliant subject (No. 6; nCPAP $3 \mathrm{~h} \cdot$ night $^{-1}$ ) had a deterioration in ANS test score: 1 pre-nCPAP to 1.5 post-nCPAP. The improvement in ANS test score in the five compliant patients was positively correlated with an improvement in mean $\mathrm{Sa}_{\mathrm{a}} \mathrm{O}_{2}$ during sleep posttreatment. We conclude that successful treatment of obstructive sleep apnoea leads to normalization of impaired autonomic stress responses.
\end{abstract}

Eur Respir J., 1996, 9, 2122-2126.

*Dept of Respiratory Medicine, ${ }^{+}$Sleep Grenoble, and University Joseph Fourier, Grenoble, France.

Correspondence: P.A. Lévy

Secteur Sommeil et Respiration Service de Pneumologie

CHU 38043 Grenoble Cedex 9

France

Keywords: Autonomic nervous system autonomic stress tests

nasal continuous positive airway pressure obstructive sleep apnoea syndrome

Received: January 31996

Accepted after revision May 231996

This study was supported by a grant from Région Rhone Alpes (Hypoxia).
Obstructive sleep apnoea syndrome (OSAS) is a disorder in which repetitive apnoeas during sleep produce hypoxaemia, arousals and fragmentation of sleep [1]. Apnoea-related hypoxaemia produces activation of the sympathetic nervous system [2]. The repeated acute changes in sympathetic activity lead to a chronic stimulation of the sympathetic nervous system, with increased plasma and urinary catecholamines, which can be normalized by tracheostomy [2]. There is a hypertensive response at the end of an apnoea and a further incremental elevation following the resumption of ventilation and arousal [3]. The sensitivity of the baroreceptor reflexes are altered during apnoeas in hypertensive OSAS [4]. A possible mechanism could be chronic autonomic nervous system (ANS) stimulation, as occurs with the recurrent blood pressure (BP) surges during apnoeas. CARLSON et al. [5] have shown persistent increase in muscle sympathetic nerve activity even during the awake period in obstructive sleep apnoea (OSA) patients. Thus, chronic ANS stimulation may alter autonomic responses in OSA.

We have previously shown that OSA is associated with abnormalities in autonomic stress tests in relation to overnight oxygen desaturation (cumulative time with $\mathrm{Sa}_{\mathrm{a}} \mathrm{O}_{2}$ $<90 \%$ and minimal $\mathrm{Sa}_{\mathrm{a}} \mathrm{O}_{2}$ in sleep) [6]. We have postulated that treatment of OSA may ameliorate this dysfunction. We have, therefore, studied ANS function pre- and post-treatment with nasal continuous positive airway pressure (nCPAP).

\section{Patients}

We studied six males who had been referred for polysomnography because of suspected OSA, with severe snoring. All of these patients had moderate to severe obesity at the time of the study, with a mean body mass index (BMI) of $32.2 \mathrm{~kg} \cdot \mathrm{m}^{-2}$ (range $24.5-39.2 \mathrm{~kg} \cdot \mathrm{m}^{-2}$ ), and were aged $43-58$ yrs (mean $52 \pm 5 \mathrm{yrs}$ ). One patient received a diuretic preparation, which was stopped $48 \mathrm{~h}$ before each examination. This was the only patient with diagnosed hypertension.

Subjects were excluded from the study if they had angina or a history of recurrent tachycardia. Diabetes mellitus and current consumption of beta-blockers, alphaagonists, vasodilators or angiotensin converting enzyme 
(ACE) inhibitors, or cholinergic stimulating drugs also led to exclusion. Patients were informed of the methods and aims of the study and consent was given to the protocol, which was approved by the Hospital Ethics Committee. This was in agreement with the Declaration of Helsinki on Clinical Experiments.

Patients completed a questionnaire about symptoms and previous illnesses, which was used for the clinical assessment of those with suspected sleep-disordered breathing. They were asked about hypersomnolence, snoring and morning headache. They were also questioned about symptoms relating to autonomic dysfunction, such as postural hypotension and impotence. The subjects underwent a clinical examination and BP was recorded lying at rest during the afternoon as the baseline. This BP value was compared to that recorded on waking in the morning.

\section{Methods}

\section{Polysomnography}

Polysomnography was performed in each patient twice: once for diagnosis and once for assessment of progress under nCPAP. Polysomnographic recording included electroencephalogram (EEG), with electrode positions C3/A2-C4/A1-CZ/O1 of the international 10-20 electrode placement system; electro-oculogram (EOG), chin electromyogram (EMG) and electrocardiogram (ECG, modified V2 lead) were also recorded. Respiration was monitored with abdominal and thoracic strain gauges. Airflow was semiquantitatively measured by the sum of buccal and nasal thermistors, and $\mathrm{Sa}_{\mathrm{a}} \mathrm{O}_{2}$ was measured with a Biox-Ohmeda 3700 oximeter.

The polysomnogram was scored manually, according to standard criteria [7]. Episodes of apnoea were defined as complete cessation of airflow for $10 \mathrm{~s}$ or more, and hypopnoea as a greater than $50 \%$ decrease in oronasal airflow lasting for at least $10 \mathrm{~s}$. Apnoea/hypopnoea events were classified as central, obstructive or mixed, according to the presence of breathing efforts. The OSAS was defined by the respiratory disturbance index (RDI, i.e. number of episodes of apnoea/hypopnoea $\cdot \mathrm{h}^{-1}$ of sleep) [8].

\section{Autonomic function tests [9, 10]}

Tests were performed in the following order:

Heart rate response to Valsalva manoeuvre. The subject was instructed to blow maximally through a mouthpiece attached to a manometer. He lay semirecumbent and had an ECG recorded at a paper speed of $25 \mathrm{~mm} \cdot \mathrm{s}^{-1}$. He was instructed to blow hard for $15 \mathrm{~s}$, maintaining the manometer at the maximal value, and then suddenly to release his breath. This was repeated three times. The R-R intervals of the ECG were examined and the ratio of the longest R-R during release to the shortest R-R interval during strain calculated (Valsalva ratio).

Heart rate response to deep breathing. This was performed in the supine position. The subject took a slow deep breath over $5 \mathrm{~s}$ and exhaled over $5 \mathrm{~s}$ for a period of 1 min (i.e. 6 breaths $\cdot \mathrm{min}^{-1}$ ) whilst the ECG was recorded.
The ratio (E/I) of the mean of the maximum R-R intervals during deep expiration (six breaths) to the mean of the minimum R-R intervals during deep inspiration (six breaths) was calculated.

Heart rate response to standing up. A continuous ECG was recorded, whilst the subject rose suddenly from the supine to the upright position. R-R intervals of beats 15 and 30 after standing were recorded and a 30/15 ratio calculated.

Postural response in blood pressure. BP was recorded by mercury or aneroid sphygmomanometer, with the subject lying after $5 \mathrm{~min}$ rest. The subject was instructed to stand up quickly and the BP recorded immediately. The difference in systolic blood pressure (SBP) was calculated ("postural response").

Blood pressure response to sustained handgrip. The subject was instructed to squeeze a dynamometer to a pressure of $30 \%$ of their maximum for $3 \mathrm{~min}$. The BP was measured at $30 \mathrm{~s}$ intervals with a mercury or aneroid sphygmomanometer. The change in diastolic blood pressure (DBP) was recorded ("handgrip response").

Tests were performed by two doctors experienced in the use of these autonomic stress tests. The patient rested between each test in order for the heart rate to return to baseline. Patients were studied on the day of polysomnography at 17:00-18:00 $\mathrm{h}$ in the sleep laboratory, after $1 \mathrm{~h}$ sitting at rest and without cigarettes or coffee consumption. Tests were performed at 06:00 h the following morning as the patient woke naturally from sleep.

\section{Analysis of results}

The tests were analysed by two of the doctors without knowledge of the polysomnographic data, and marginal results were reviewed by the third doctor without knowledge of the previous result.

The normal values of INGALL et al. [10] for ECG responses to the various manoeuvres were used, as these take into account the age of the subject. The normal values for BP responses were those of EwING [9], which consider a postural fall of $<30 \mathrm{mmHg}$ in SBP as normal. A rise of $10-15 \mathrm{mmHg}$ in DBP in response to handgrip is marginally abnormal, and $<10 \mathrm{mmHg}$ rise definitely so.

Since the test results are defined as normal or abnormal for different actual values in relation to age, each abnormal test was scored as +1 and each marginal result as +0.5 .

One patient refused to have autonomic tests performed on the morning of his study under nCPAP and, thus, the evening results pre- and post-nCPAP were compared in this case.

Compliance was assessed by reading the clock counter in the nCPAP machine. Quantitative variables were compared using a Wilcoxon signed rank test and a Chisquared analysis with Yates' correction for comparison of qualitative variables.

\section{Results}

The patients were studied after at least 1 year of treatment with nCPAP, with a median of 471 (389-624) days treatment (table 1). All of the patients were symptomatic 
Table 1. - Patient demographics and ANS test results which were abnormal or marginal

\begin{tabular}{lccccccc}
\hline $\begin{array}{l}\text { Pt } \\
\text { No. }\end{array}$ & $\begin{array}{c}\text { Age } \\
\text { yrs }\end{array}$ & $\begin{array}{c}\text { BMI } \\
\text { pre- } \\
\text { nCPAP } \\
\mathrm{kg} \cdot \mathrm{m}^{-2}\end{array}$ & $\begin{array}{c}\text { BP } \\
\text { pre- } \\
\text { nCPAP } \\
\text { mmHg }\end{array}$ & $\begin{array}{c}\text { ANS Tests } \\
\text { Pre- } \\
\text { nCPAP }\end{array}$ & $\begin{array}{c}\text { ANS Tests } \\
\text { on } \\
\text { nCPAP }\end{array}$ \\
\hline 1 & 56 & 29 & $134 / 92$ & $2,4,5(4.5)$ & 5 & $(0.5)$ \\
2 & 50 & 35 & $130 / 70$ & 1,3 & $(3)$ & 5 & $(0.5)$ \\
3 & 50 & 25 & $118 / 80$ & 2 & $(1)$ & 0 & $(0)$ \\
4 & 55 & 35 & $120 / 80$ & 2,5 & $(1)$ & 0 & $(0)$ \\
5 & 43 & 36 & $150 / 100$ & 2,5 & $(1)$ & 0 & $(0)$ \\
6 & 58 & 27 & $140 / 100$ & 2 & $(1)$ & 2 & $(1.5)$ \\
\hline
\end{tabular}

Pt: patient; BMI: body mass index; nCPAP: nasal continuous positive airway pressure; ANS: autonomic nervous system. 1= Valsalva response; $2=$ deep breathing response; $3=1$ lying/standing heart rate response; 4=postural blood pressure response; $5=$ handgrip blood pressure response. The ANS test scores for morning plus evening are shown in parentheses.

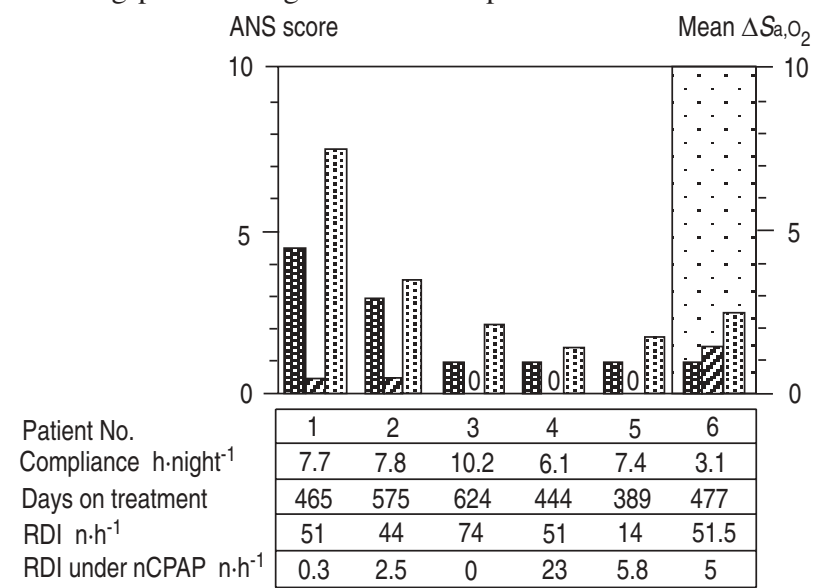

Fig. 1. - Autonomic scores (left axis) and $\Delta \mathrm{Sa}_{\mathrm{a}, \mathrm{O}_{2}}$ (right axis) and patient clinical details. Hospital polysomnography pre- and undernCPAP treatment. Note deterioration in ANS score in noncompliant patient (No. 6;,$\cdot$ ). 䁒 : ANS score before nCPAP; $\mathbb{Z}$ : ANS score post-nCPAP; : mean nocturnal $\Delta S_{\mathrm{a}, \mathrm{O}_{2}}$ (pre-under nCPAP). nCPAP: nasal continuous positive airway pressure; ANS: autonomic nervous system; $\Delta S_{a}, \mathrm{O}_{2}$ : difference in arterial oxygen saturation; RDI: respiratory disturbance index.
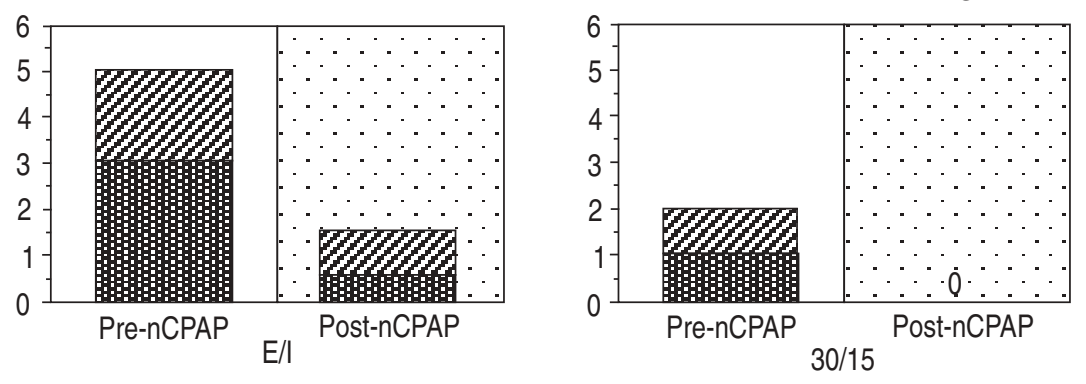

from their OSA before treatment but none complained of any autonomic-related symptoms prior to or after nCPAP. There was no significant change in mean body mass index (BMI) over the treatment period $(31 \pm 5$ and $33 \pm 6 \mathrm{~kg} \cdot \mathrm{m}^{-2}$ post-treatment, respectively). The patients were generally compliant with treatment using nCPAP for a mean of $7.1 \pm 2.4 \mathrm{~h} \cdot$ night $^{-1}$, but one patient used his treatment for only $3 \mathrm{~h} \cdot$ night $^{-1}$ on average (fig. 1). The patients had significant OSA, with a respiratory disturbance index (RDI) of $48 \pm 19$ (range 14-74) events $\cdot h^{-1}$ pretreatment. On the night of polysomnography with nCPAP, five out of six patients had normalization of the RDI to $<10$ events $\cdot \mathrm{h}^{-1}$ of sleep. One patient had only a partial response on the night of study on nCPAP, with an improvement in RDI from 51-23 events $\cdot \mathrm{h}^{-1}$. His mean $\mathrm{Sa}_{\mathrm{a}} \mathrm{O}_{2}$ pre-nCPAP was $92.5 \%$ and ANS score was one. With treatment, mean $\mathrm{Sa}_{\mathrm{a}} \mathrm{O}_{2}$ improved to $94 \%$ and ANS score was normal.

Blood pressure values in the morning pre-nCPAP are shown in Table 1. One patient (No. 6), who was noncompliant with treatment, had a deterioration in total evening plus morning scores of autonomic stress tests after nCPAP. His score increased to 1.5 in the second phase of the study, having been 1.0 pre-nCPAP (fig. 1). The five compliant patients all had a reduction in ANS score: total score for the five pre-nCPAP (morning and evening) was 10.5 out of a possible maximum score of 45 , and post-nCPAP this fell to 1.0 out of 45 . This was a significant change $(\mathrm{p}<0.05)$. As in a previous report [1], we examined whether the level of oxygenation overnight had any relationship to the ANS test scores. We found that the change in ANS test score correlated with the change in mean $\mathrm{Sa}_{\mathrm{a}} \mathrm{O}_{2}$ overnight between diagnosis and treatment $(\mathrm{r}=0.97 ; \mathrm{p}<0.01)$.

The overnight changes in SBP $(131 \pm 14.5$ vs $132 \pm 12.1$ $\mathrm{mmHg})$ and DBP $(89.8 \pm 8.6$ vs $87.6 \pm 12.7 \mathrm{mmHg})$ in the pretreatment phase were not significant. This was also the case after nCPAP treatment (SBP $132 \pm 17$ vs $133 \pm 23.9$ mmHg and DBP $84 \pm 19$ vs $90 \pm 10 \mathrm{mmHg}$ overnight).

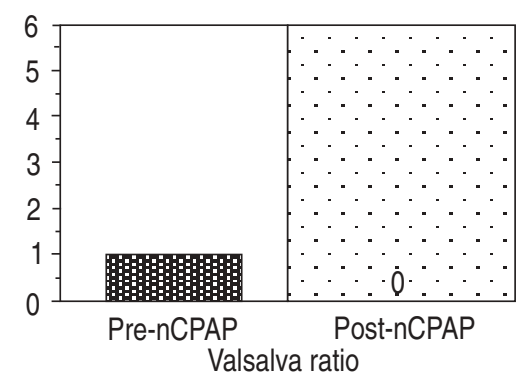

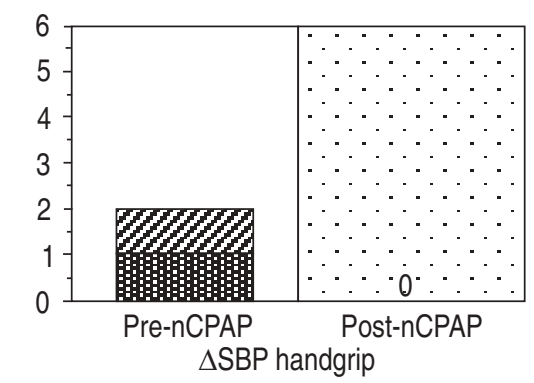

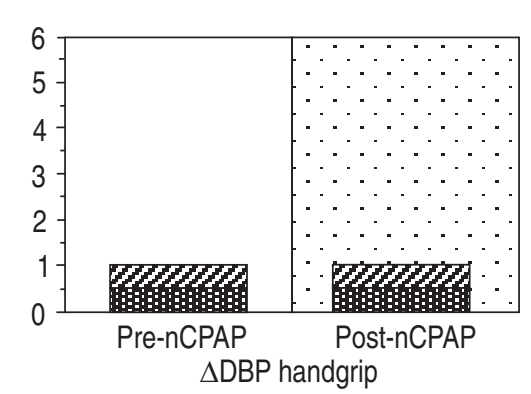

Fig. 2. - Evolution of each ANS test score before and after nCPAP summarized for all of the patients. Note that the majority of the abnormalities were found in the heart-rate stress test. 䀠抯 ANS stress tests evening; $\mathscr{Z} Z$ : ANS stress tests morning; $\square$ : ANS stress test before nCPAP; : ANS stress tests after nCPAP. E/I: ratio of the mean of the maximum R-R intervals during deep expiration (six breaths) to the mean of the minimum R-R intervals during deep inspiration (six breaths); 30/15: ratio of intervals of beats 15 and 30 after standing. $\triangle$ SBP: change in systolic blood pressure; $\triangle \mathrm{DBP}$ : change in diastolic blood pressure. For further definitions see legend to figure 1. 
The noncompliant patient had a relative increase of $\mathrm{SBP}$ to $165 \mathrm{mmHg}$ during the second study in the morning.

Figure 2 shows that the E/I test was the most often abnormal, but only one patient had an abnormal result for this test on nCPAP. The majority of abnormalities in response were in heart rate tests.

\section{Discussion}

We have previously demonstrated abnormalities of ANS stress tests in patients with OSA [6]. We have now shown that effective treatment of this condition by nCPAP can lead to an improvement in these abnormal stress responses.

We acknowledge that these findings relate to a small group of patients but the results were consistent. Ideally, a control group of subjects should be studied but this would have to be carried out in a set of patients with milder OSA, as it would not be justifiable to leave patients with an average AHI of 48 events $\cdot h^{-1}$ without effective therapy for more than a year.

The greatest level of abnormality was in the response to deep breathing. It may be that patients with OSA systematically experience increased stimulation of their mechanoreceptors during each apnoea, with large changes in pulmonary volume. This may indicate that the heart rate response to respiratory efforts could be chronically reset in relation to the abnormal respiratory events at night over the years of development of OSA.

Likewise, the chronic sympathetic discharge in these patients could change autonomic responses before treatment. EsCOURRou et al. [4] showed that the sensitivity of the baroreflex in sleep apnoea syndrome (SAS) subjects while awake, assessed by the slope of the response between heart rate and BP on injection of phenylephrine, was normal in normotensive SAS subjects but reduced in hypertensive SAS subjects. Furthermore, normotensive SAS patients had a greatly increased sensitivity in baroreflex during apnoeas, which was not evident in hypertensive SAS. In the present study, the more important changes, however, were in the parasympathetic mediated responses.

Acute autonomic effects, such as abrupt swings in heart rhythm from bradycardia to tachycardia [11], raised serum and urine levels of catecholamines [2], and muscle sympathetic nerve activity bursts [12] have been described in OSA. Furthermore, chronic diurnal modifications of autonomic function have been found in this condition, with raised sympathetic tone [12] and hypertension [13]. Fletcher et al. [2] have shown that raised overnight cat-echolamine secretion can be reversed by tracheostomy. HEDNER et al. [14] showed that an increased level of sympathetic activity can be reduced by 14-26 months nCPAP, but this did not reduce blood pressure or cardiac structure as measured by left ventricular mass index [14]. In this study, it was demonstrated that more long-term autonomic effects of OSA can be reversed by nCPAP. This suggests that adrenergic tone as well as ANS stress test responses can be normalized, whilst arterial and cardiac effects may persist.

The present patients were examined after at least 1 year of nCPAP treatment, so the rate of change in autonomic function with improvement in OSA is not known.
Patients who benefit from nCPAP generally have a rapid improvement in symptoms, such as daytime somnolence [15] but the course of longer term evolution of autonomic effects, such as improvements in BP, have not been reported, although nCPAP treatment may improve BP levels [16]. We did not find an overnight fall in BP, which would be an expected consequence of a normalization of sympathetic tone with proper treatment of OSA. Continuous monitoring of BP may better detect evolutionary changes in BP.

There has been much discussion as to which battery of cardiovascular reflex tests is best suited for assessing autonomic responses and what constitutes normal response values [17-19]. In the present study, the tests of EwING [9] were used as the basis of the selection of tests, as these have been in use for many years and are wellestablished. However, as a middle-aged group of patients were studied, age-related normal values were taken for the heart rate responses [10].

In our previous study [6], the level of failure of autonomic response to stress was related to a measure of the severity of overnight oxygen desaturation and, indeed, in the present report the level of improvement in autonomic stress response was related to the level of improvement in $S \mathrm{a}, \mathrm{O}_{2}$. The only patient with inadequate improvement in RDI had a clear improvement in mean nocturnal $\mathrm{Sa}_{\mathrm{a}} \mathrm{O}_{2}$ to a value of $94 \%$. The fact that we have shown that the degree of ANS stress response dysfunction and its improvement both seem to relate to the degree of overnight oxygen desaturation would seem to indicate that hypoxia has a role to play. STEWART et al. [20] have shown that the level of abnormalities in ANS stress tests is also related to the level of hypoxia in chronic obstructive pulmonary disease (COPD). There are, however, no studies relating this to sleep structure or catecholamine secretion.

We acknowledge that other mechanisms, such as microarousal or repeated respiratory effort against a closed airway, might be implicated in the deterioration of autonomic responses and relieved by nCPAP. Somers et al. [21] recently showed that increased sympathetic nerve activity was reduced by nCPAP in OSA patients. Further studies are needed to elucidate the precise mechanisms of changes in autonomic responses.

The management of OSA by nCPAP treatment has been revolutionized but, unfortunately, it is not tolerated by all patients, mainly because of mask-related nasal problems [22]. Noncompliance may be as high as $20 \%$ and, thus, it is not surprising that one of the present patients was documented as using his machine for only 3 h.night ${ }^{-1}$. The fact that this patient had a deterioration in ANS test score adds strength to the argument that proper treatment of OSA may reverse some of the ill effects of the disease. Admittedly, this patient had an improvement in overnight $\mathrm{Sa}_{\mathrm{a}} \mathrm{O}_{2}$ on the night of polysomnography under nCPAP but this was with $7 \mathrm{~h}$ of effective treatment. It was not known what the effect of intermittent therapy at a mean of $3 \mathrm{~h} \cdot$ night $^{-1}$ might be. BARONE KRIBBS et al. [23] showed that even one night without nCPAP can be associated with the recurrence of sleeprelated breathing disturbance and daytime somnolence. Thus, the deterioration in our noncompliant patient, although minimal, supports the idea that ANS dysfunction is secondary to OSA and only reversible with adequate treatment. 
In COPD, it has been shown that autonomic dysfunction is related to prolongation of the QTc interval on the electrocardiogram [24], and to an increased mortality risk. Prolonged QTc interval puts the patient at risk of serious arrhythmias [25]. However, FLEMONS et al. [26] did not find an increased level of dysrhythmia in OSA patients overnight compared to nonapnoeic snorers. GILLIS et al. [27] showed that the QTc was prolonged during the onset of apnoea but shortened significantly during apnoea and postapnoea hyperventilation. A study focused on a subgroup of apnoeic patients with autonomic dysfunction might identify patients with prolonged QTc interval, who could, thereby, be at risk of arrhythmia.

We conclude that impaired autonomic responses occur in obstructive sleep apnoea and that these are probably related to nocturnal hypoxia, which can be improved by nasal continuous positive airway pressure. Further study of the effects of obstructive sleep apnoea and its treatment on autonomic control may help elucidate the mechanisms of development of systemic effects in this condition.

\section{References}

1. Guilleminault C, Dement WC. Sleep apnea syndromes. New York, Alan R. Liss Inc., 1978.

2. Fletcher EC, Miller J, Schlaf JW, Fletcher JG. Urinary catecholamines before and after tracheostomy in obstructive sleep apnea and hypertension. Sleep 1987; 10: $35-44$.

3. Shepard JW. Haemodynamics in Obstructive Sleep Apnea. In: Fletcher EC, ed. Orlando, Grune and Stratton, 1986; pp. 39-61.

4. Escourrou P, Le Gros V, Gaultier C. Baroreflex control of heart rate in sleep apnea patients: comparison of normotensive and hypertensive patients and effect of CPAP therapy. In: Gaultier C, Escourrou P, Curzi-Dascalova L, eds. Sleep and Cardiorespiratory Control. Colloque INSERM. Vol. 217. 1991; pp. 193-201.

5. Carlson J, Hedner J, Elam M, Ejnell H, Sellgren J, Wallin G. Augmented resting sympathetic activity in awake patients with obstructive sleep apnea. Chest 1993; 103: 1763-1768.

6. Veale D, Pepin JL, Levy PA. Autonomic stress tests in obstructive sleep apnea syndrome and snoring. Sleep 1992; 15: 505-513.

7. Rechtschaffen A, Kales A. A manual of standardized terminology, techniques and scoring system for sleep stages of human subjects. US Department of Health, Education and Welfare. Publication No. (NIH) 204 National Institute of Health, 1968.

8. American Thoracic Society. Indications and standards for cardiopulmonary sleep studies. Am Rev Respir Dis 1989; 139: 559-568.

9. Ewing DJ. Cardiovascular reflexes and autonomic neuropathy. Clin Sci Mol Med 1978; 55: 321-327.
10. Ingall TJ, McLeod JG, O'Brien PC. The effect of ageing on autonomic nervous system function. Aust NZ J Med 1990; 20: 570-577.

11. Guilleminault C, Connolly S, Winkle R, Melvin K, Tilkian A. Cyclical variation of the heart rate in sleep apnoea syndrome. Lancet 1984; i: 126-131.

12. Hedner J, Ejnell H, Sellgren J, Hedner T, Wallin G. Is high and fluctuating muscle nerve sympathetic activity in the sleep apnea syndrome of pathogenic importance for the development of hypertension? J Hypertens 1988; 6: S529-531.

13. Working group on OSA and hypertension. Obstructive sleep apnea and blood pressure elevation: what is the relationship? Blood Pressure 1993; 2: 166-182.

14. Hedner J, Darpo B, Ejnell H, Carlson J, Caidahl K. Reduction in sympathetic activity after long-term treatment in sleep apnoea. Eur Respir J 1995; 8: 222-229.

15. Rajagopal KR, Bennett AL, Dillard TA, Telis CJ, Tenholder MF. Overnight nasal CPAP improves hypersomnolence in sleep apnea. Chest 1986; 90: 172-176.

16. Mayer J, Becher H, Brandenburg U, Penzel T, Peter JH, von Wichert P. Blood pressure and sleep apnea: results of long-term nasal continuous positive airway pressure therapy. Cardiology 1991; 79: 84-92.

17. Ryder REJ, Hardisty CA. Which battery of cardiovascular autonomic function tests? Diabetologia 1990; 33: 177-179.

18. Wieling W, van Lieshout JJ. The assessment of cardiovascular reflex activity: standardisation is needed. Diabetologia 1990; 33: 182-183.

19. Ewing DJ. Which battery of cardiovascular autonomic function tests? Diabetologia 1990; 33: 180-181.

20. Stewart AG, Waterhouse JC, Howard P. Cardiovascular autonomic nerve function in patients with hypoxaemic chronic obstructive pulmonary disease. Eur Respir J 1991; 4: 1207-1214.

21. Somers VK, Dyken ME, Clary MP, Abboud FM. Sympathetic neural mechanisms in obstructive sleep apnea. J Clin Invest 1995; 96: 1897-1904.

22. Pepin JL, Léger P, Veale D, Langevin B, Robert D, Lévy $P$. Side-effects of nasal continuous positive airway pressure in sleep apnea syndrome (study of 193 patients in two French sleep centres). Chest 1995; 107: 375-381.

23. Barone Kribbs N, Pack AL, Kline LR, et al. Effects of one night without nasal CPAP treatment on sleep and sleepiness in patients with obstructive sleep apnea. Am Rev Respir Dis 1993; 147: 1162-1168.

24. Stewart AG, Waterhouse JC, Howard P. The QTc interval, autonomic neuropathy and mortality in hypoxaemic COPD. Respir Med 1995; 89: 79-84.

25. Algra A, Tijssen JGP, Roelandt JRTC, Pool J, Lubsen J. QTc prolongation, measured by standard 12-lead ECG, is an independent risk factor for sudden death due to cardiac arrest. Circulation 1991; 83: 1888-1894.

26. Flemons WW, Remmers JE, Gillis AM. Sleep apnea and cardiac arrhythmias: is there a relationship? Am Rev Respir Dis 1993; 148: 618-621.

27. Gillis AM, Stoohs R, Guilleminault C. Changes in the QT interval during obstructive sleep apnea. Sleep 1991; 14: 346-350. 